\title{
Endogenous siRNAs and piRNAs derived from transposable elements and genes in the malaria vector mosquito Anopheles gambiae
}

\author{
Inna Biryukova ${ }^{1^{*}}$ and Tao Ye ${ }^{2}$
}

\begin{abstract}
Background: The siRNA and piRNA pathways have been shown in insects to be essential for regulation of gene expression and defence against exogenous and endogenous genetic elements (viruses and transposable elements). The vast majority of endogenous small RNAs produced by the siRNA and piRNA pathways originate from repetitive or transposable elements (TE). In D. melanogaster, TE-derived endogenous siRNAs and piRNAs are involved in genome surveillance and maintenance of genome integrity. In the medically relevant malaria mosquito Anopheles gambiae TEs constitute 12-16\% of the genome size. Genetic variations induced by TE activities are known to shape the genome landscape and to alter the fitness in An. gambiae.

Results: Here, using bioinformatics approaches we analyzed the small RNA data sets from 6 libraries formally reported in a previous study and examined the expression of the mixed germline/somatic siRNAs and piRNAs produced in adult An. gambiae females. We characterized a large population of TE-derived endogenous siRNAs and piRNAs, which constitutes $56-60 \%$ of the total siRNA and piRNA reads in the analysed libraries. Moreover, we identified a number of protein coding genes producing gene-specific siRNAs and piRNAs that were generally expressed at much lower levels than the TE-associated small RNAs. Detailed sequence analysis revealed that An. gambiae piRNAs were produced by both "ping-pong" dependent (TE-associated piRNAs) and independent mechanisms (genic piRNAs). Similarly to D. melanogaster, more than $90 \%$ of the detected piRNAs were produced from TE-associated clusters in An. gambiae. We also found that biotic stress as blood feeding and infection with Plasmodium parasite, the etiological agent of malaria, modulated the expression levels of the endogenous siRNAs and piRNAs in An. gambiae.

Conclusions: We identified a large and diverse set of the endogenously derived siRNAs and piRNAs that share common and distinct aspects of small RNA expression across insect species, and inferred their impact on TE and gene activity in An. gambiae. The TE-specific small RNAs produced by both the siRNA and piRNA pathways represent an important aspect of genome stability and genetic variation, which might have a strong impact on the evolution of the genome and vector competence in the malaria mosquitoes.
\end{abstract}

Keywords: endo-siRNAs, endo-piRNAs, Anopheles gambiae, Transposable elements

\section{Background}

Small non-coding RNAs (ncRNAs) are involved in regulation of gene expression, RNA based immunity and activity of transposable elements (TEs) and their remnants. In D. melanogaster two small RNA silencing pathways, small interfering RNAs (siRNA) and piRNA

\footnotetext{
* Correspondence: inbiryukova@gmail.com

'Department of Vector Biology, Max Planck Institute for Infection Biology (MPIIB), Berlin 10117, Germany

Full list of author information is available at the end of the article
}

(PIWI-interacting RNAs), regulate gene expression by silencing specific genes and TEs both in germline and soma [1-7]. In Dipteran insects siRNAs represent a population of small RNA 21-nucleotides (nt) in length. siRNA is originated from exo- and endogenous double stranded RNA (dsRNA), which is processed by the RNAse III enzyme Dicer-2 (Dcr-2) into 21-nt siRNA duplexes. Once produced and loaded into RNAinduced silencing complex (RISC), siRNAs guide in sequence-specific manner the recognition and cleavage

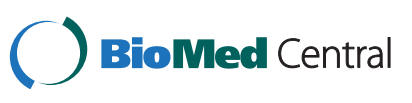


of the target RNA molecules by the Argounate-2 (Ago-2) containing RISC. Similarly to D. melanosgaster, the Ago-2 expression in mosquitoes is required for antiviral defence. Depletion of Ago-2 in An. gambiae and Ae. aegypti mosquitoes respectively infected with O'nyong-yong virus and Sindbis virus resulted in increased virus replication $[8,9]$.

piRNAs represent a population of small RNAs ranging from 24 to $30-n t$ in length, that are specifically expressed in gonads of metazoans and provide genome integrity over generations [10-12]. In insects the major source of piRNAs is transposable genetic elements that are considered as selfish or parasitic elements of the host genome. piRNAs post-transcriptionally silence TE expression through the piRNA-guided cleavage of the transposon mRNA [13]. In addition, piRNAs can mediate transcriptional control of TE activity [6]. The piRNA-mediated RNA silencing requires an association with Ago family members Ago-3, Aubergine (Aub) and PIWI (P-element induced wiped testis) that operate at the heart of the piRNA silencing pathway [13-15]. In contrast to siRNAs, piRNAs are produced in Dicer-independent manner from a long single-stranded RNA transcribed from repetitive elements or genomic loci known as piRNA clusters. This transcript is processed into primary piRNAs that are usually antisense to TE transcript. These primary piRNAs direct to cleave sense TE transcripts and initiate a reciprocal amplification cycle so-called "pingpong" amplification loop [13,15]. PIWI-class members exhibit strand specific interaction with piRNAs, PIWI and Aub are associated with antisense TE-piRNAs; while Ago-3 is associated with sense TE-piRNAs $[13,15]$. The hallmarks of the ping-pong mediated amplification of piRNAs are strong U1 bias for Aub-associated piRNAs and A10 of Ago-3-associted piRNAs. In flies, Aub and Ago-3 are not expressed in the ovarian somatic sheet (OSS) cell line and as a result primary piRNAs lack a ping-pong signature, yet showing strong bias for $5^{\prime} \mathrm{U}$ [16]. In addition to the function of piRNAs in controlling TE activity, the piRNA pathway is involved in antiviral response in D. melanogaster and mosquito cell lines [4,17-22]. Altogether, the piRNA-mediated pathway constitutes an adaptive immune response that recognizes and silences invading parasitic genetic elements [15,23].

TEs represent a large part of dispersed repetitive elements, recognized as one of the major cause of intra-genomic variation and genome diversification. The vertically transmitted TEs can disperse rapidly into populations [24]. De novo TE remobilization and insertion are capable of significantly influencing genome stability, host gene transcription, splicing or RNA editing. Subsequent TE invasion, dispersion, inactivation and deterioration forms a unique genome landscape and represents a process of "molecular domestication" of mobile elements [25]. TEs spread over genome via an RNA or a
DNA intermediate and have been classified accordingly into two classes, class I (retrotransposons) and class II (DNA transposons). Class I is composed of long terminal repeat (LTR) retrotransposons and non-LTR (NLTR) retrotransposons that are structurally similar to retroviruses and require a reverse transcription step for retrotransposition. Class II TEs includes DNA transposons with i) cut-and-paste mechanism of transposition, ii) rolling-circle DNA transposons (Helitrons) and iii) self-synthesizing DNA transposons (Polintrons) [26]. In An. gambiae, TEs constitute about $12-16 \%$ of the euchromatic regions and more than $60 \%$ of the heterochromatic regions of the genome [27,28]. Overall more than 350 different transposon families have been identified in the genome of An. gambiae, the most abundant are LTR-retrotransposons, short interspersed nuclear elements (SINEs) and miniature inverted transposable elements (MITEs) superfamilies [24,27,29].

It has been reported that hematophagous arthropod vector mosquitoes produce virus-derived small RNAs in the soma in response to viral infection [19,20,30,31]. Furthermore, these mosquitoes produce endogenous siRNAs and piRNAs that map to TEs and protein coding genes $[31,32]$. The precise function of endogenously produced siRNAs and piRNAs remains largely unknown in mosquito species. Both the siRNA and piRNA pathways represent an important aspect of genetic variation, which might have a strong impact on evolution of the host genome landscape and influence the fitness and vector competence in the malaria mosquito. Using previously reported deep sequenced small RNA libraries [33] we examined the expression of endogenous siRNAs and piRNAs produced in adult An. gambiae females. We identified a large set of TE- and gene-associated siRNAs and piRNAs, which might be involved in the regulation of transposable element activity and gene expression in soma and germline in An. gambiae. Detailed sequence analysis revealed that $A n$. gambiae piRNAs were produced by both "ping-pong" dependent and "ping-pong" independent mechanisms. We also found that regular and infectious blood feeding modulated the expression levels of the endogenous small RNA populations and the core components of the siRNA and piRNA pathways in An. gambiae.

\section{Results}

\section{Small RNA sequencing}

We performed analysis of small RNA populations recovered from the deep sequenced small RNA libraries published in [33] to determine i) diversity of the mixed germline/somatic endogenous siRNAs and piRNAs in $A n$. gambiae adult females ii) how endogenous siRNAs and piRNAs respond to blood feeding and Plasmodium berghei infection. We used a combined strategy [16,32,34-36] 
following the annotation of piRNAs and siRNAs by analysing sequence signature characteristics of piRNAs/siRNAs and performing a validation by a homology-based analysis of the identified sequences to known piRNA and siRNA loci in D. melanogaster and Ae. aegypti. We filtered out the known miRNAs, rRNAs, tRNAs and snoRNAs (miRBase, VectorBase, Rfam) from the libraries for further analysis. The reference strain, PEST (AgamP3.8, VectorBase), which segregates into two different haplotypes (S and $\mathrm{M}$ ) for certain regions of the genome [27] was used to map small RNA reads to the An. gambiae genome. The analysed genome of the susceptible $A n$. gambiae G3 strain (S haplotype) is divergent from the PEST genome, therefore we allowed 2-nt mismatching for mapping. The analysis of the small RNA populations ranging from 15 to 44-nt in length from sugar, blood and $P$. berghei-infected mosquito libraries, revealed that sequences of 20-23-nt lengths (a 21-nt peak length) and 2430-nt (a 27-nt peak length) were predominantly recovered (Figure 1A-B; Additional file 1: Table S1). Sequences in the 21-nt and 24-30-nt size range, not annotated as a previously known non-coding RNAs, and passing the cut-off at least 10 read uniquely mapped per individual endosiRNA or endo-piRNA were classified accordingly as candidate siRNAs and piRNAs. Additionally to the 21-nt and 27-nt peak lengths we also detected a large number of other small non-coding RNAs peaking at $16 \mathrm{nt}$ and $23 \mathrm{nt}$ (Figure 1A-B, Additional file 2: Table S2 and Additional file 3: Table S3). Analysis of the An. gambiae genome complexity revealed that protein coding genes constitute $7 \%$ of the genome, TEs $-12-16 \%$ and total intergenic regions $\sim 78 \%$ [27]. We found the following distribution of 21 -nt reads in sugar-fed mosquito libraries, $12.6 \%$ mapped to the protein coding genes, $31 \%$ mapped to intergenic regions and $56.4 \%$ mapped to repetitive and transposable elements (Figure 1C; Additional file 2: Tables S2 and Additional file 3: Tables S3). The 24-30-nt read distribution showed a similar tendency mapping to protein coding genes (16\%) and intergenic regions (24\%); 60\% were originated from repetitive elements and TEs (Figure 1C; Additional file 2: Tables S2 and Additional file 3: Tables S3). Moreover, we observed a similar frequency for genic 21-nt and 24-30-nt reads in mosquitoes after regular blood feeding (Figure 1C), whereas TE-associated number of reads was increased relatively to the levels in sugar-fed females. Interestingly, we also noticed higher density of the TE-associated and genic 21-nt and 24-30-nt reads in mosquitoes after infectious blood feeding (Figure 1C). Overall, in our libraries more than 55\% of all 21-nt and 24-30-nt reads mapped to known TEs (Figure 2A,B). Importantly, TE-associated 24-30-nt reads exhibited a strong bias towards the antisense strand recapitulating the sequence signatures of piRNAs produced via ping-pong dependent piRNA pathway (Figures 2 and 3). A similar pattern for piRNAs has been previously reported for D. melanogaster and Ae. aegypti
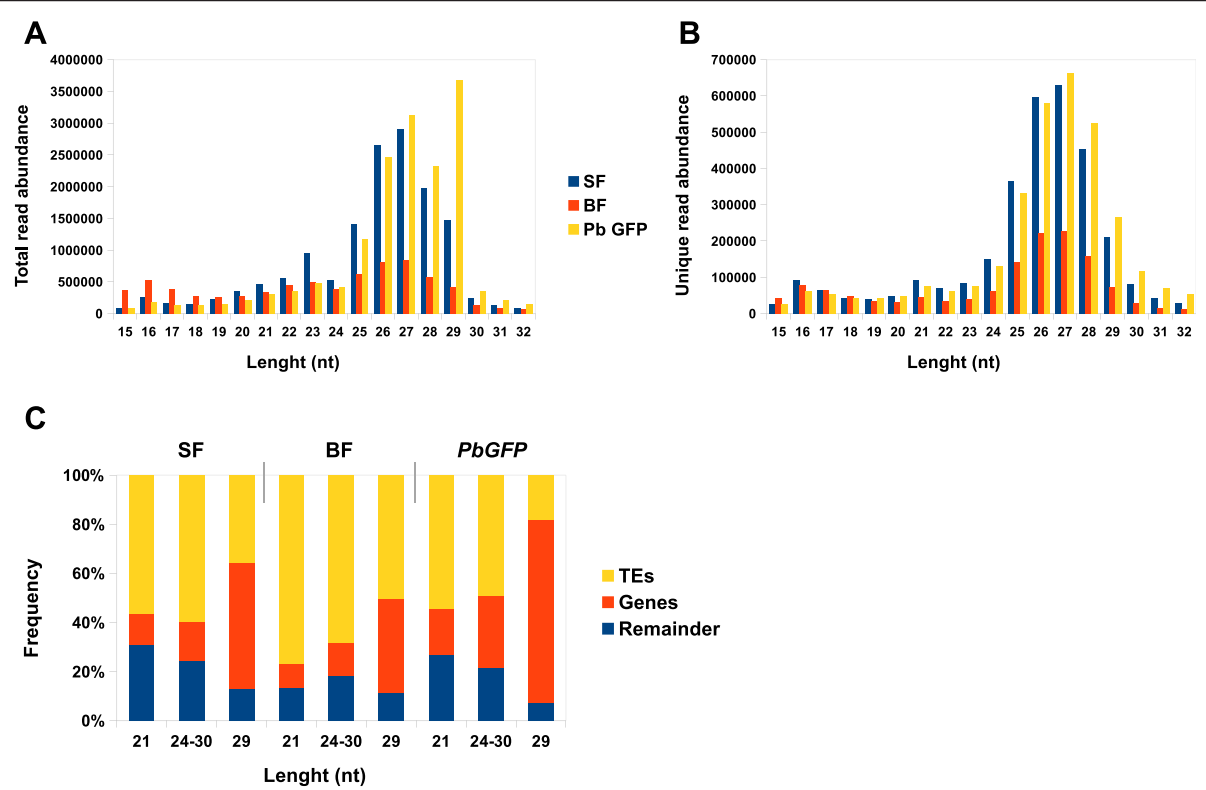

Figure 1 Sequencing summary of siRNA and piRNA populations in An. gambiae. Size distribution for the total number of sequence reads (A) and for unique sequence reads (B) in An. gambiae CDNA libraries derived from sugar (SF), blood-fed (BF) and P. berghei infected (PbGFP) mosquitoes. The cDNA libraries from replicate were collapsed; the length and abundance of small RNA reads in An. gambiae cDNA libraries are as indicated. (C) Read frequency for siRNAs (peaking at $21 \mathrm{nt}$ ) and piRNAs (peaking at 24-30 nt) mapped to repetitive elements, consisting largely of TEs, coding genes and other sequences (remainder) in An. gambiae cDNA libraries. 
A

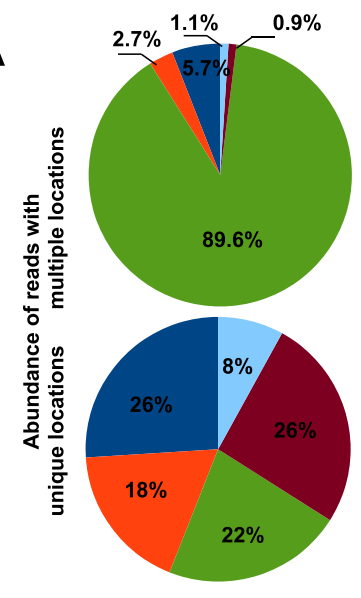

$21 \mathrm{nt}$

n LTR retrotransposons

- NLTR retrotransposons

SINEs

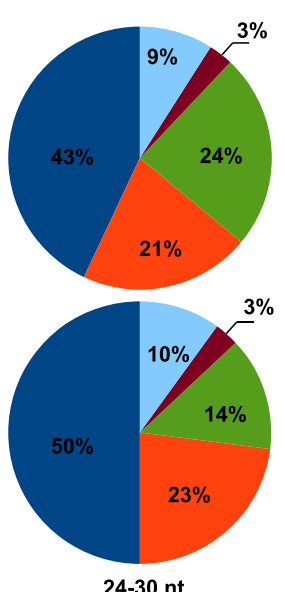

24-30 nt
B

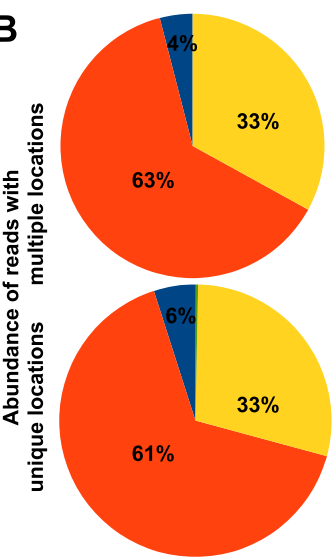

21nt

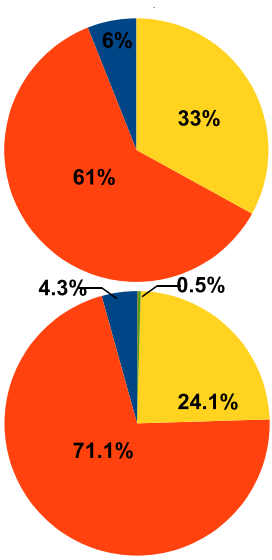

24-30 nt

DNA transposons

uknown transposons

C

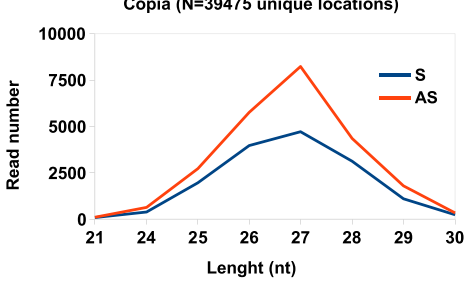

D

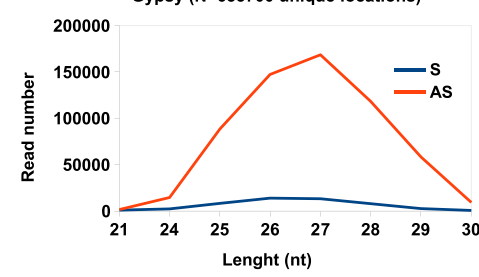

E

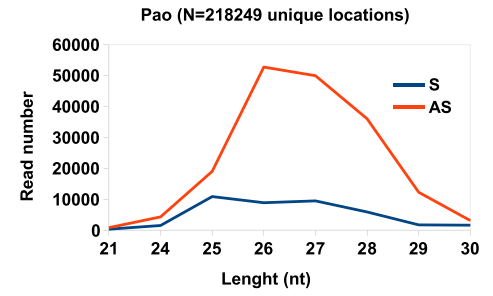

F

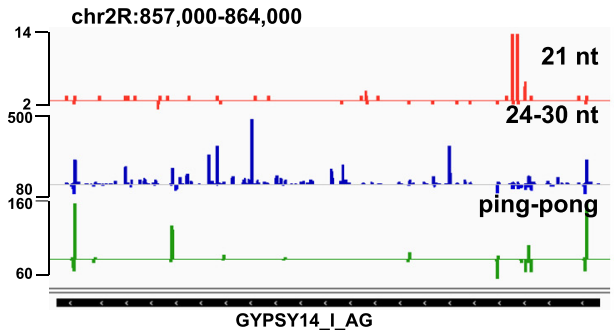

Copia ( $\mathrm{N}=87210$ multiple locations)

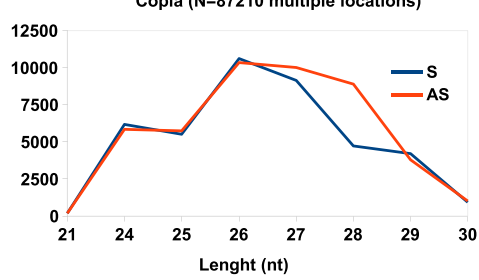

Gypsy ( $N=467611$ multiple locations)
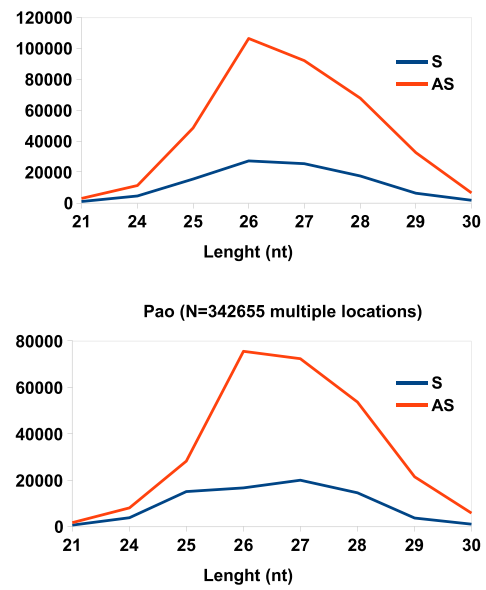

G

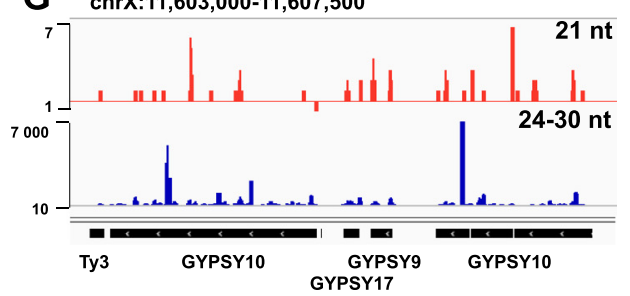

Figure $\mathbf{2}$ (See legend on next page.) 
(See figure on previous page.)

Figure 2 TE-piRNAs and TE-siRNAs in An. gambiae. (A) Read frequency for a siRNA population (21 nt) and a piRNA population (24-30 nt) associated with various classes of TEs in An. gambiae (EnsemblMetazoa). (B) Read frequency for piRNAs mapped to class I superfamilies (LTR and NLTR TES). (C-E) Small RNA read frequency and distribution mapped to various families of TEs. Distribution of reads uniquely mapped to An. gambiae genome (left) and reads mapped to the genome five and more times (right). N indicates the number of reads; sense reads (S) and antisense reads (AS) are shown as indicated. (F-G) Genomic profile of the 21-nt (red), 24-30-nt (blue) and piRNA "ping-pong" paired (green) reads mapped to the sense and antisense strand of gypsy transposon (represented by an abundantly expressed single full-length copy (F) and a cluster of truncated and Solo-LTR copies (G)) in the An. gambiae genome.

[15,32]. Furthermore, some An. gambiae endo-piRNAs matched to known Ae. aegypti piRNAs [20,32] indicating identification of bona fide piRNAs.

\section{TE-specific siRNA and piRNA expression in An. gambiae}

Repetitive and transposable elements represent the major source of endogenous piRNAs and siRNAs in Dipteran insects and other animals [13,15,37-40]. The genome landscape of An. gambiae is represented by members of both classes, class I (retrotransposons) and class II (DNA transposons) $[24,27,29]$, which constitute $6.85 \%$ and $5.9 \%$ respectively of the total An. gambiae genome size $[27,32,41]$. We analysed genomic distribution and density of endosiRNAs (21-nt) and endo-piRNAs (24-30-nt) sequence reads mapped to the collection of repetitive and mobile elements from the genome of An. gambiae using EnsemblMetazoa database in sugar-fed mosquitoes (Figure 2A-E). Mapping of 21-nt reads to the class I and class II of TEs revealed that $10.4 \%$ and $86 \%$ of total endo-siRNA reads were associated with retrotransposons and DNA transposons respectively. Besides, $\sim 67 \%$ of the total endo-piRNA reads (24-30-nt) were derived from retrotransposons and $21 \%$ from DNA transposons. The distribution of the small RNA reads derived from unique genomic loci was consistent with the distribution of the multiply mapped 24-30-nt reads (Figure 2A); moreover, the uniquely mapped 21-nt reads showed a redistribution of read frequencies (26\%, $18 \%, 22 \%$ for the LTR, NLTR retrotransposons and DNA transposons, respectively) compared with the total and multiply mapped 21-nt reads (Figure 2A).

Recent analysis of the TE distribution revealed that class I constitutes two-third and class II - one-third of the total TEs identified in the genome of An. gambiae (34\%, 33\%, 33\% for the LTR, NLTR retrotransposons and DNA transposons, respectively) [24]. Therefore, we further analysed in detail the class I LTR and NLTR-derived siRNAs and piRNAs (Figure 2B-G). The class-I LTR-TEs are represented by three retrotransposon superfamilies: copia ( $\geq 800$ copies), Pao-Bel ( 1000 copies) and gypsy ( $\geq 1000$ copies) [27]. They constitute approximately $4 \%, 35 \%$ and $52 \%$ respectively of the total TE number in the genome of An. gambiae [29]. The distribution of the total 24-30-nt read frequency revealed that $5.6 \%$ of TE-piRNAs were associated with copia, $30 \%$ with $\mathrm{Pao}-\mathrm{Bel}$ and $64 \%$ with gypsy, indicating that copia, Pao-Bel and gypsy were likely the most actively transcribed LTRretrotransposons in our libraries. Next, we analysed the density and distribution of uniquely and multiply mapped 21-nt and 24-30-nt reads derived from these LTR-TEs (Figure 2C-E; Additional file 4: Figure S1). The pool of multiply mapped 21-nt and 24-30-nt (five genomic location and more) were considered respectively as siRNAs and piRNAs derived from dispersed copies of the LTRTEs. We found that copia produced uniquely mapped siRNAs and piRNAs with a bias towards antisense orientation (Figure 2C). Besides, among multiply mapped piRNAs, only class of 27-28-nt reads exhibited a mild antisense bias. gypsy and Pao-Bel generated a high proportion of uniquely and multiply mapped piRNA reads with a strong antisense bias (Figure 2D-E). In D. melanogaster gypsy produced abundantly both classes of TE-associated small RNAs, siRNAs and piRNAs [16]. An. gambiae gypsy abundantly produced piRNAs, predominantly $26-27$-nt reads in length and a very low number of siRNAs unlike Drosophila gypsy (Figure 2D,F-G). gypsy elements represent the most abundant and diverse LTR superfamily in the $A n$. gambiae genome $[24,41]$. In total, $42 \%$ and $15 \%$ of gypsy copies represent remnants of the gypsy full-length transposon, so-called solitaire Solo-LTR and truncated copies respectively [24]. The sense and antisense distribution of 21-nt and 24-30-nt reads along the sequence of gypsy revealed a multiple hot spots of gypsy-specific siRNAs and piRNAs that were spread along the TE sequence and LTRrs (Figure 2F-G).

Class-I NLTR-retrotransposons is composed of 14 superfamilies in the An. gambiae genome [24,42]. The NLTRs represent the most abundant and diverse type of TEs in An. gambiae [24]. We noticed that the total abundance of NLTR-piRNAs and siRNAs was low relative to its size (Figure 2A,B) probably due to extremely low proportion of full-length elements in NLTRs in comparison to LTRs [24]. Jockey, CR1, RTE and Outcast elements represent the most abundant superfamilies (15\%,63\%, $11 \%$ and $7 \%$ respectively of the total NLTR-TEs in the genome of An. gambiae; [29]). We found that $44 \%$ of the total NLTR-piRNA reads were derived from Jockey, CR1, RTE and Outcast retrotransposons in the analysed small RNA libraries (Additional file 2: Table S2). We also observed that the SINEX-1 family, which represents a large part of the total NLTR-TEs [24] was associated 




with a low number of siRNA and piRNA reads in our small RNA libraries (Additional file 4: Figure S1).

In the An. gambiae genome, DNA transposons are represented by most heterogeneous sets of heavily deteriorated sequences or their remnants in the analysed class II families. Overall, the MITE-like elements, which are lacking any coding capacity, represent $60 \%$ of the class II transposons [24]. The most abundant class II-superfamilies are Tc1-Mariner (35\%), P element (10\%), hAT (8\%), Harbinger (4\%) and novel unknown elements $\sim 32 \%$ [29]. In our small RNA libraries, members of Harbinger, Tc1-Mariner, P element, Gambol elements and unknown TE104184 transposon were the major sources of 21-nt reads and 24-30-nt reads derived from class II DNA transposons (Additional file 4: Figure S1).
Overall, we observed that transcriptional activities of the class I-derived siRNAs and piRNAs correlate with the abundance of the LTR- and NLTR-retrotransposons (gypsy, Pao-Bel, copia; CR1, Jockey, Outcast and RTE) in our small RNA libraries (Figure 2A-B, Additional file 4: Figure S1). We also noticed a similar tendency for the class II-derived siRNAs (mapped to Harbinger, Tc-1/ Mariner, $P$ element and Gambol superfamily). Besides, the total abundance of the DNA transposon-derived piRNAs was low relative to the size of class II DNA transposons in the An. gambiae genome.

Next we analysed the relative nucleotide frequencies in the An. gambiae TE-siRNAs. The sequence analysis revealed a bias for $5^{\prime}$-end $C$ at position 1 in the total 21 -nt reads and in the 21-nt reads that mapped to sense strand 
of TEs (Figure 3A,C). A similar bias was observed in $D$. melanogaster endogenous siRNAs that often began with $\mathrm{C}$ [40]. In addition, we noticed a bias for $5^{\prime} \mathrm{U}$ at position 1 for 21-nt reads derived from antisense TE strand (Figure 3A,C). The nucleotide composition across individual D. melanogaster TE families showed 5' $\mathrm{U}$ bias in 21-nt reads mapped to antisense strand and no bias in 21 -nt reads mapped to sense strand [16,40], this is consistent with the $5^{\prime} \mathrm{U}$ preference for the 21-nt antisense reads mapped to specific TEs in An. gambiae (results not shown). Next we performed an overlap analysis of sense-antisense 21-nt paired reads. We used (+19-nt) offset to detect 21-nt siRNA duplex with the 3'-end 2-nt overhangs produced by RNAse III enzyme Dcr-2 (Figure 3B). The nucleotide composition analysis revealed a bias at position 1 for $5^{\prime} \mathrm{C} / \mathrm{G}$ in sense and $5^{\prime} \mathrm{C}$ in antisense strand (Figure 3C). In $D$. melanogaster TEs produce siRNAs in the almost equivalent ration between sense and antisense orientation with a slight antisense bias [16]. Unlike in Drosophila, in our small RNA datasets the An. gambiae TE-siRNAs showed a strong sense bias. The bias was introduced by the 21-nt reads mapped to unknown TE104184 transposon (Additional file 4: Figure S1), which produced the vast majority of 21-nt sense reads in our libraries. TE104184 belongs to class II DNA transposons (EnsemblMetazoa) and is represented by more than 800 copies in the An. gambiae genome. Non-random distribution and precision of the $5^{\prime}$ - and 3 '-ends of the TE104184-derived 21-nt reads ruled out the possibility that these reads might represent the incidental degradation fragments (Additional file 4: Figure S1).

In contrast to the An. gambiae TE-siRNAs, the TEpiRNAs exhibited a strong over-presentation of antisense reads with $5^{\prime} \mathrm{U}$ bias (Figure $3 \mathrm{C}$ ). We analysed in details the occurrence of sense-antisense piRNA arrangements and their nucleotide composition for i) non-paired neighbour piRNAs, ii) overlapped piRNA ping-pong pairs and iii) overlapped piRNA ping-pong pairs with phased arrangement considering a plausible under-representation in our small RNA libraries using 0 -nt; +10-nt and (-16-18-nt) offsets respectively ([16], Figure 3B). There was a $5^{\prime} \mathrm{U}$ bias for antisense TEpiRNA reads in all analysed offsets and a strong bias for $\mathrm{A}$ at position 10 for reads derived from sense strand in +10-nt and (-16-18-nt) offsets (Figure 3B). These results implied that vast majority of the TE-specific piRNAs exhibited the classical hallmarks of ping-pong amplification and most likely were produced by the ping-pong dependent mechanism in An. gambiae.

Given the functional association of the core factors of the siRNA pathway (Dcr-2 and Ago-2) and piRNA pathway (PIWI-class transcripts PIWI, Aub and Ago-3) in TE silencing, we analysed expression of the most abundant
LTR-retrotransposon, gypsy and NLTR-retrotransposon, CR1 in RNA silenced adult female mosquitoes (Additional file 5: Figure S2). The An. gambiae PIWI family genes Ago-3 (AGAP008862), PIWI2/Aub (AGAP009509) and PIWI1/Aub (AGAP011204) were identified by sequence similarity with the D. melanogaster Ago homologs [43,44]. RNAi directed against Dcr-2/Ago-2 and PIWI-class genes reduced their transcript levels approximately $30-50 \%$ according to the qPCR-based measurement (Additional file 5: Figure S2). We observed consistent mild desilencing of CR1 in PIWI1-Aub silenced mosquitoes, indicating RNAi-dependent degradation. Besides, expression of the gypsy element was not substantially affected in RNA silenced mosquitoes and was varied significantly between biological replicates (Additional file 5: Figure S2) probably due to variegating expression of numerous truncated copies as a result of some subtle differences in mosquito rearing condition.

\section{Genic siRNA and piRNA loci in the genome of An. gambiae}

Given that 9-29\% of 21-nt and 24-30-nt reads mapped to protein coding genes in our small RNA libraries (Figure 1C; Additional file 3: Tables S3), we analysed the association between protein coding genes and small RNA sequences (Figure 4) in order to better understand the role of endo-piRNAs and endo-siRNAs. Distribution of the total 21-nt and 24-30-nt reads that mapped to coding genes showed a sense bias for genic piRNAs and siRNAs (Figure 4A-B). The overlap analysis of siRNA pairs revealed almost equivalent sense and antisense distribution in 21-nt reads with a mild antisense bias for (+19 nt) offset. The 24-30-nt piRNA paired reads showed a slight sense bias in the $0 \mathrm{nt}$ and +10 nt offsets, whereas antisense bias (almost two-fold) was detected for (-16-18 nt) offset piRNA pairs (Figure 4B). In addition, we observed consistently $5^{\prime}$ A preference for antisense 21-nt reads and $5^{\prime} \mathrm{U}$ for antisense 24-30-nt reads. We also noticed presence of A at position 10 for total 24-30-nt reads in sense orientation (Figure 4B). However, this signature was missing in the $(+10 \mathrm{nt})$ offset paired sense reads, suggesting a ping-pong independent mode of piRNA biogenesis for piRNAs produced by coding genes. Importantly, we did not find in the siRNA pool derived from the protein coding gene any "ping-pong" like signatures meaning that they do not belong to a subset of the piRNA-like small RNAs. It is worth noting that we also observed a production of endogenous piRNAs and siRNAs from intergenic regions in An. gambiae genome (Figure 3A). The intergenic 21-nt and 24-30 nt reads also showed a strong $5^{\prime} \mathrm{U}$ and 5' U/A bias respectively (Figure 3A).

Next we analysed the frequency and distribution of the most abundant 21-nt reads and 24-30-nt reads mapped to specific An. gambiae genes (Figure 4C-D; 

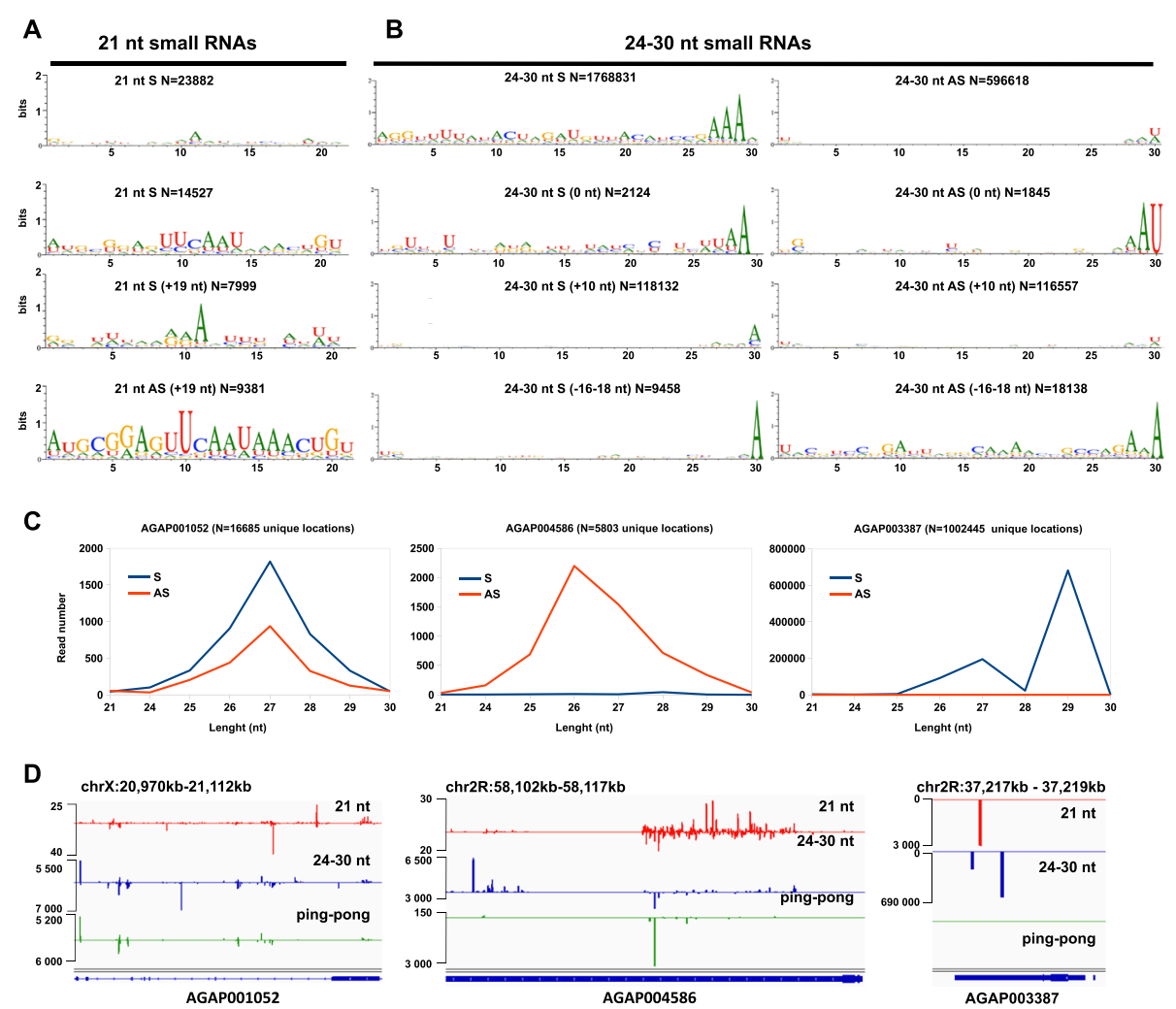

Figure 4 Sequence characterization of gene-specific piRNAs and siRNAs in An. gambiae. Sequence properties of sense and antisense siRNA (A) and piRNA (B) populations mapped to protein coding genes in An. gambiae (VectorBase). The offsets for siRNA and piRNA pairs are shown in (Figure 3B). N indicates the number of reads. (C) Distribution of unique matching reads mapped to AGAP001052, AGAP004586 and AGAP003387 (as representative hot spots of 21-nt and 24-30-nt reads); sense reads (blue); antisense reads (red). (D) Genomic profiles showing density of the 21-nt (red), 24-30-nt (blue) and piRNA "ping-pong" paired (green) reads mapped to the sense and antisense strand of AGAP001052, AGAP004586 and AGAP003387.

Additional file 4: Figure S1). The analysis of small RNA density revealed that two genes AGAP006442 and AGAP003387 produced one-third of total 21-nt reads in our libraries (Additional file 3: Tables S2 and Additional file 4: Tables S3; Additional file 4: Figure S1). The AGAP006442-associated 21-nt reads mapped to both sense and antisense strands (Additional file 4: Figure S1), whereas AGAP003387-derived 21-nt reads were predominantly in sense orientation. Among the top abundant small RNA producing genes, AGAP001052 produced 21nt and 24-30-nt reads in almost equivalent proportion from both strands with a sense bias (Figure 4C,D); whereas AGAP004586-associated 21-nt reads and 24-30nt reads showed a strong antisense bias (Figure 4C,D). Both AGAP001052 and AGAP004586 were producing paired 24-30-nt reads with "ping-pong" signatures (Figure 4D). The most abundant genic source of 21-nt and 24-30-nt reads in our libraries was AGAP003387, which encodes a protein with unknown function and has a single ortholog in A. quadriannulatus - AQUA010285, putative lipoprotein (VectorBase). Interestingly more than $50 \%$ of 24-30-nt reads in sugar- and blood-fed mosquitoes and more than $75 \%$ in $P$. berghei-infected mosquitoes were associated with AGAP003387 (Additional file 3: Table S3). This gene produced small RNA reads predominantly in a sense orientation including two classes of the most abundant reads, 27-nt and 29-nt in length (Figure 4C). Genomic profiling of 21-nt and 24-30-nt reads that mapped to AGAP003387 showed asymmetric distribution with the highest read density at the $3^{\prime}$ end (Figure 4D). Importantly, the ping-pong piRNA pairs were not detected within the AGAP003387-derived population of 24-30-nt reads (Figure 4D). The $3^{\prime}$ end piRNA profile associated with AGAP003387 might be similar to the profile of genes producing piRNAs from their 3' UTR in D. melanogaster $[36,45,46]$.

The vast majority of endogenous siRNAs and piRNAs originate from transcription of TEs, cis-natural antisense transcripts (cis-NAT) or long inverted repeat transcripts (structured hairpin RNAs). The genomic organization of AGAP006442 and AGAP003387, which produced the most abundant genic siRNAs, does not show any $3{ }^{\prime}$-end transcript overlapping (VectorBase). Similarly to those, AGAP004586 is a stand-alone gene, no other known 
RNA transcripts within a $10 \mathrm{~kb}$ genomic window has been annotated (VectorBase), yet we cannot rule out the existence of unknown cis-NAT transcripts in the analysed genomic region. AGAP001052 exhibits convergent arrangement with its neighbour gene showing tail-to-tail organization with AGAP001051. The most abundant $A n$. gambiae endo-siRNAs were not associated with convergent transcripts, similarly to Ae. aegypti, which produced the majority of endo-siRNAs from non-overlapping regions (50\%) and only 3.8\% from tail-to-tail adjacent transcripts [31]. Interestingly, the detailed examination of the An. gambiae loci producing the most abundant classes of genic small RNA reads revealed that $80 \%$ of the analysed protein coding genes were framed by nested or clustered repetitive elements or TEs in the surrounding distal and proximal regions.

We also analysed the effect of RNA silencing of PIWIclass and Dcr-2/Ago-2 genes on the AGAP003387 and AGAP001052 transcript levels using qPCR. The expression level of AGAP001052 mRNA was not associated with a significant desilencing; whereas, level of AGAP003387 transcript was increased in PIWI-class silenced mosquitoes (Additional file 5: Figure S2). Correlation analysis of the genic 3' UTR piRNAs revealed that highly expressed transcripts tend to generate more piRNAs [36]. Curiously, the increased level of AGAP003387 transcript in blood-fed and infected mosquitoes did not correlate with piRNA levels in our libraries measured at $3 \mathrm{~h}$ after feeding (Additional file 3: Table S3, Additional file 6: Figure S3). However, the transcriptional level of AGAP003387 detected 24 h after infectious blood feeding was significantly lower than after a regular blood feeding (Additional file 6: Figure S3). This might reflect the delayed kinetics of the piRNA-mediated silencing.

Multiple negative feedbacks regulating the core components of small RNA silencing pathways have been reported $[1,31,32,36,40]$. To identify siRNAs and piRNAs involved in negative feedback circuits in the siRNA and piRNA networks in An. gambiae, we analysed 21-nt and 24-30-nt reads that mapped to the main components of the siRNA and piRNA pathways (Table 1). We noticed that the core components of the siRNA biogenesis Dcr2, its cofactors $R 2 D 2$ and Loqs-2 were associated with extremely low read numbers (10 and less reads) mapped to the sense strand. We also observed a similar tendency for the RNA helicase Spindle E (spnE), DEAD-box helicase DDX17 (Rm62) and Rm68, which are known to be implicated in RNA biogenesis, microRNA and viral RNA processing in Drosophila $[47,48]$. In the An. gambiae genome four putative $R m 62$ orthologs were predicted by OrthoDB; among them only AGAP003663 was associated with a substantial number of 24-30-nt reads mapped in a sense orientation (Table 1). Interestingly, the core components of the microRNA pathway, which produces endogenous small RNAs involved in repression of partially complementary mRNAs, Drosha, Pasha and Dcr-1 were not enriched for 21-nt or 24-30-nt reads (Table 1). Besides two main effectors of the RISC, Ago-2 (siRISC) and Ago-1 (miRISC) were associated with higher density of 21-nt and 24-30-nt reads in a sense orientation.

In addition, the core components of the piRNA biogenesis were not associated with genic siRNAs and piRNAs in our libraries (Table 1). The exception was AGAP005672, one of the Tudor-domain containing orthologues that are known to be involved in the regulation of various small RNAs. Two genes, traffic jam ( $t j)$ and maelstrom (mael), have been reported to be implicated in the piRNA biogenesis and piRNA production in D. melanogaster and in Ae. aegypti [32,45]. Unlike Ae aegypti and D. melanogaster, the An. gambiae orthologs of $t j$ and mael showed extremely low frequency for the associated genic 21-nt and 24-30-nt reads. Overall, we observed that in adult An. gambiae mosquitoes i) genic-siRNAs were expressed at much lower levels than TE-siRNAs in all analysed libraries, ii) genic piRNAs were generally lacking ping-pong sequence signatures and most likely produced by "ping-pong" independent mechanism, iii) the core components of the siRNA and piRNA biogenesis were under avoidance of the negative feedback regulatory loop.

\section{Clustering siRNAs and piRNAs in the genome of $A n$. gambiae}

In Drosophila, the majority of piRNAs ( 92\%) derive from uni- and dual-strand piRNA clusters that occupy only $3.5 \%$ of the fly genome and consist largely of transposon sequences [15]. The genomic loci producing clustering piRNAs are often associated with clustering siRNAs [40,49]. We analysed the genomic piRNA and siRNA cluster distribution in collapsed sugar-fed mosquito libraries by scoring for clustering of the uniquely and multiply mapped 21-nt reads and 24-30-nt reads (Methods; Table 2, Additional file 7: Table S5). We found that the chromosome 2 was highly enriched for total abundance of both uniquely and multiply mapped clustering piRNAs and siRNAs (Figure 5, Additional file 7: Table S5). A detailed analysis of transcriptional activity of the clustering small RNAs revealed that the most abundant piRNA and siRNA clusters were associated with TE clusters on chromosomes $\mathrm{X}$ and $2 \mathrm{R}$, respectively (Figure 5; Additional file 7: Table S5). In Drosophila the most abundant piRNA cluster size ranged from 2 to 242 $\mathrm{kb}$ and in Aedes mosquitoes - from 6 to $184 \mathrm{~kb}[15,32]$. In $A n$. gambiae the size of the most abundant genic piRNA and TE-piRNA clusters was $170 \mathrm{~kb}$ and $520 \mathrm{~kb}$ respectively (Additional file 7: Table S5), suggesting that An. gambiae piRNA clusters cover greater genomic distances then in the genome of $D$. melanogaster or Ae. 
Table 1 Genic siRNAs and piRNAs associated with the core components of the small RNA silencing pathways in An. gambiae

\begin{tabular}{|c|c|c|c|c|}
\hline \multicolumn{3}{|l|}{ Small RNA pathway } & $\begin{array}{l}\text { siRNAs } \\
\text { 21-nt read number }\end{array}$ & $\begin{array}{l}\text { piRNAs } \\
\text { 24-30-nt read number }\end{array}$ \\
\hline \multirow[t]{4}{*}{ siRNA } & Loqs-2 & AGAP009781 & 0 & 0 \\
\hline & $R 2 D 2$ & AGAP009887 & 2 & 3 \\
\hline & Dcr-2 & AGAP012289 & 3 & 8 \\
\hline & Ago-2 & AGAP011537 & 84 & 251 \\
\hline \multirow[t]{16}{*}{ piRNA } & Ago-3 & AGAP008862 & 0 & 18 \\
\hline & Aub-PIWI2 & AGAP009509 & 2 & 61 \\
\hline & Aub-PIWI1 & AGAP011204 & 9 & 93 \\
\hline & Zucchini & AGAP006233 & 1 & 0 \\
\hline & Armitage & AGAP006939 & 12 & 44 \\
\hline & Tudor & AGAP005672 & 20 & 391 \\
\hline & Vreteno & AGAP010722 & 1 & 0 \\
\hline & Shutdown & AGAP011458 & 0 & 19 \\
\hline & Pimet/HEN1 & AGAP005646 & 0 & 15 \\
\hline & $t j$ & AGAP010030 & 1 & 8 \\
\hline & mael & AGAP002022 & 2 & 3 \\
\hline & spnE & AGAP002829 & 11 & 9 \\
\hline & $R m 62(D D X-17)$ & AGAP003663 & 29 & 773 \\
\hline & & AGAP004912 & 1 & 6 \\
\hline & & AGAP005652 & 7 & 93 \\
\hline & & AGAP012045 & 14 & 104 \\
\hline \multirow[t]{4}{*}{ miRNA } & Dcr-1 & AGAP002836 & 1 & 5 \\
\hline & Drosha & AGAP008087 & 0 & 0 \\
\hline & Pasha & AGAP002554 & 1 & 2 \\
\hline & Ago-1 & AGAP011717 & 122 & 412 \\
\hline
\end{tabular}

aegypti. We also noticed that piRNA clusters produced more than $90 \%$ of the detected piRNAs in An. gambiae; similarly to $84 \%$ of the observed piRNAs produced by the piRNA clusters in A. agypti [32]. In An. gambiae, clustering TE-piRNAs predominantly mapped to the antisense strand, whereas genic clustering piRNAs were in sense orientation (Additional file 7: Table S5). In addition, clustering siRNAs were mapped preferentially to the sense strand of both, protein coding genes and TEs in our libraries.

A number of studies in An. gambiae have reported that blood meal intake induces massive changes in transcript levels $3 \mathrm{~h}$ after feeding compared to nonblood fed mosquitoes [50]. To determine how endogenous siRNAs and piRNAs respond to blood feeding and $P$. berghei infection, we analysed small RNA expression in mosquitoes $3 \mathrm{~h}$ after regular and infectious blood feeding. We quantified and assessed the differential expression of 21-nt and 24-30-nt reads in our libraries using the DESeq2.6 package [51]. We detected very few siRNAs and piRNAs with statistically significant changes in their levels in $P$. berghei-infected and blood-fed mosquitoes (Additional file 8: Table S6). It has been shown that biotic stress triggered by pathogen infection can induce de novo production of endogenous siRNAs and piRNAs in mosquitoes [20,30,31]. In our analysis $P$. berghei infection was not associated with de novo production of TE-siRNAs or TE-piRNAs. However, blood feeding induced more than ten-fold changes in the expression levels of the under-represented (<10 reads) 21nt and 24-30-nt reads mapped to DNA transposons (Additional file 8: Table S6). Additionally, we detected only a few de novo produced genic piRNAs and siRNAs in the presence of $P$. berghei and a few genic piRNAs were induced by regular blood feeding (Additional file 8: Table S6) that became a source of additional piRNAs in adult females. Overall, we observed mild changes in the expression levels of endogenous siRNAs and piRNAs in adult blood-fed and $P$. berghei-infected mosquitoes that may represent a general stress response to blood feeding and infection. 
Table 2 The top 30 abundant TE-associated piRNA clusters in An. gambiae

\begin{tabular}{|c|c|c|c|c|c|c|c|c|c|}
\hline chr & Start & End & Length & $\begin{array}{l}\text { Unique } \\
\text { read } \\
\text { number }\end{array}$ & $\begin{array}{l}\text { Total read } \\
\text { number }\end{array}$ & $\begin{array}{l}\text { S unique } \\
\text { read } \\
\text { number }\end{array}$ & $\begin{array}{l}\text { AS unique } \\
\text { read number }\end{array}$ & $\begin{array}{l}\text { S total } \\
\text { read } \\
\text { number }\end{array}$ & $\begin{array}{l}\text { AS total } \\
\text { read } \\
\text { number }\end{array}$ \\
\hline $\operatorname{chr} x$ & 22952163 & 23481504 & 529341 & 12617 & 524714 & 5662 & 6955 & 306526 & 218188 \\
\hline chrX & 23518519 & 23797847 & 279328 & 6050 & 287157 & 3153 & 2897 & 185847 & 101310 \\
\hline $\operatorname{chrX}$ & 24283538 & 24392789 & 109251 & 5931 & 234948 & 1943 & 3988 & 67057 & 167891 \\
\hline chrX & 3290550 & 3362878 & 72328 & 4494 & 215967 & 633 & 3861 & 19123 & 196844 \\
\hline $\operatorname{chrX}$ & 21838300 & 22218800 & 380500 & 5680 & 179338 & 1955 & 3725 & 64596 & 114742 \\
\hline chrX & 11596752 & 11650633 & 53881 & 4346 & 172217 & 244 & 4102 & 6293 & 165924 \\
\hline $\operatorname{chr} X$ & 20625699 & 20944044 & 318345 & 4898 & 158982 & 1456 & 3442 & 39427 & 119555 \\
\hline $\operatorname{chr} x$ & 22577869 & 22914102 & 336233 & 4284 & 146406 & 1298 & 2986 & 40480 & 105926 \\
\hline $\operatorname{chr} x$ & 20176462 & 20391304 & 214842 & 4165 & 128553 & 1350 & 2815 & 42931 & 85622 \\
\hline $\operatorname{chr} x$ & 20970879 & 21149974 & 179095 & 3407 & 116074 & 1015 & 2392 & 34978 & 81096 \\
\hline $\operatorname{chrX}$ & 22313238 & 22549636 & 236398 & 2653 & 69651 & 875 & 1778 & 23572 & 46079 \\
\hline chrX & 20428815 & 20603496 & 174681 & 2446 & 59885 & 774 & 1672 & 18433 & 41452 \\
\hline chr2L & 89 & 481414 & 481325 & 7676 & 208890 & 2085 & 5591 & 58609 & 150281 \\
\hline chr2L & 502755 & 1019850 & 517095 & 6552 & 196698 & 1851 & 4701 & 48018 & 148680 \\
\hline chr2L & 5079181 & 5342762 & 263581 & 3826 & 140432 & 932 & 2894 & 22110 & 118322 \\
\hline chr2L & 1641056 & 1919208 & 278152 & 3433 & 90588 & 952 & 2481 & 21187 & 69401 \\
\hline chr2L & 5363783 & 5645438 & 281655 & 2881 & 86167 & 641 & 2240 & 13621 & 72546 \\
\hline chr2R & 57973180 & 58126345 & 153165 & 16504 & 712727 & 1404 & 15100 & 39319 & 673408 \\
\hline chr2R & 45825828 & 46052229 & 226401 & 3625 & 116909 & 1554 & 2071 & 48943 & 67966 \\
\hline chr2R & 60529536 & 60775476 & 245940 & 3333 & 98675 & 944 & 2389 & 21048 & 77627 \\
\hline chr2R & 60857789 & 61165860 & 308071 & 2595 & 74290 & 604 & 1991 & 14479 & 59811 \\
\hline chr2R & 54533041 & 54631947 & 98906 & 941 & 66728 & 228 & 713 & 8913 & 57815 \\
\hline chr2R & 59364672 & 59452054 & 87382 & 2003 & 64267 & 920 & 1083 & 34913 & 29354 \\
\hline chr3L & 20851509 & 20867249 & 15740 & 3210 & 186221 & 22 & 3188 & 429 & 185792 \\
\hline chr3L & 904183 & 1256919 & 352736 & 3945 & 115876 & 1341 & 2604 & 29346 & 86530 \\
\hline chr3L & 4482742 & 4823855 & 341113 & 3506 & 95767 & 1122 & 2384 & 28570 & 67197 \\
\hline chr3L & 1353818 & 1548403 & 194585 & 2203 & 75971 & 1007 & 1196 & 33069 & 42902 \\
\hline chr3L & 4891896 & 5007445 & 115549 & 1781 & 61830 & 507 & 1274 & 14210 & 47620 \\
\hline chr3L & 4238059 & 4454649 & 216590 & 1856 & 61591 & 699 & 1157 & 23730 & 37861 \\
\hline chr3R & 23627315 & 23733275 & 105960 & 2054 & 75377 & 363 & 1691 & 8532 & 66845 \\
\hline chr3R & 43053124 & 43185525 & 132401 & 2040 & 60619 & 722 & 1318 & 19578 & 41041 \\
\hline
\end{tabular}

$\mathrm{S}$ - sense orientation with respect to the genomic coordinates, AS - antisense orientation with respect to the genomic coordinates.

Expression of the core components of the PIWI and siRNA pathways in An. gambiae

While the relevance of the piRNA and siRNA pathways in adult $A n$. gambiae mosquitoes requires additional functional validation, we performed a detailed analysis of the piRNA and siRNA pathways gene expression in $A n$. gambiae. We examined expression of the core components of the piRNA (PIWI-Aub and Ago-3) and siRNA (Dcr-2 and Ago-2) pathways in adult whole body and organs composed of somatic and germline cells (Figure 6A-B). The qPCR-based measurement revealed that the PIWI-class transcripts were mainly expressed in adult gonads, ovaries and testes (Figure 6A-B), suggesting an essential and conserved role in germline development and maintenance. Besides, we did not observe any specific enrichment for Dcr-2 and Ago-2 in any of the analysed samples (Figure 6A,B,D). To determine whether the core components of the piRNA and siRNA pathways respond to blood feeding and P. berghei infection, we analysed the gene expression after regular and infectious feeding in mosquito females (Figure 6C; Additional file 6: Figure S3 and Additional file 9: Figure S4). The infectious blood feeding had only a mild effect on the transcript levels measured at $3 \mathrm{~h}$ time point (Figure 6C). Interestingly the $D c r-2$ and Ago-2 transcript levels were 


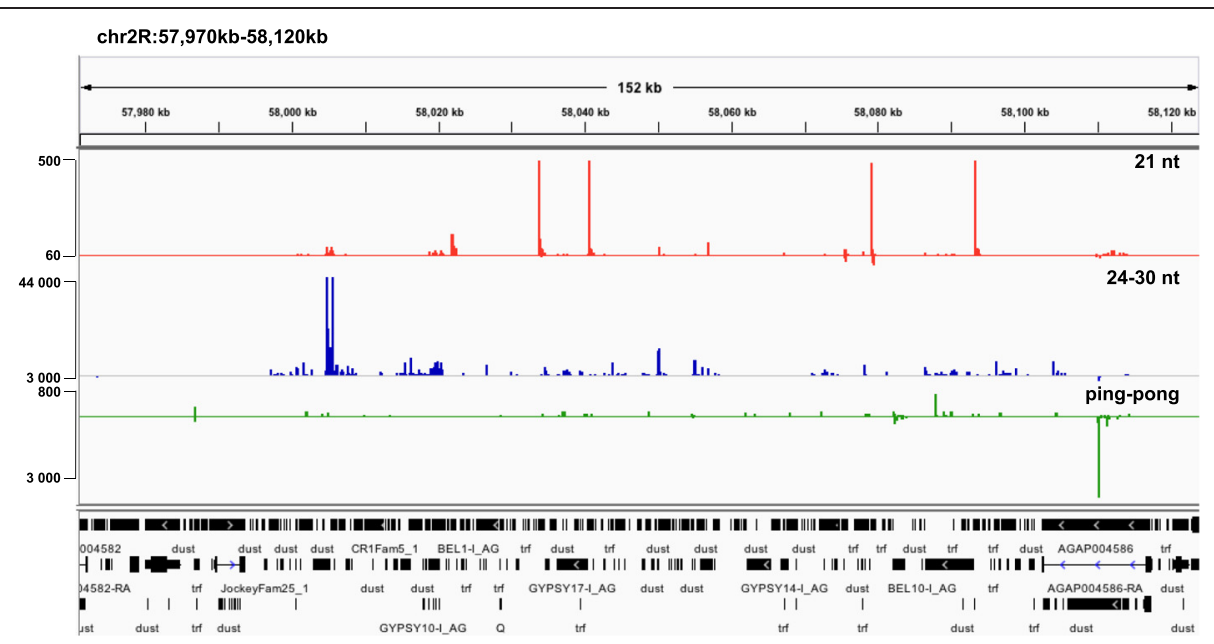

Figure 5 Pattern of clustered siRNA and piRNA transcriptional activity in An. gambiae. Graph showing hot spots of clustering siRNAs and piRNAs in respect to genomic distance in the An. gambiae genome. siRNAs (red), piRNAs (blue) and "ping-pong" piRNA pairs (green) in the clustering loci are shown as indicated.

specifically up-regulated in infected female guts (Additional file 6: Figure S3). In addition, we observed a significant upregulation of the PIWI-class transcripts $24 \mathrm{~h}$ after regular blood feeding (Figure 6C).

Given the important requirement and function of the piRNA pathway during early development, we also performed a developmental profiling of the PIWI-class genes in An. gambiae (Figure 6D). There was a significant increase in the PIWI-class transcript expression during early stages compared with adult stage we examined. We found two peaks of the PIWI-class transcript expression at embryonic and L4 stages (Figure 6D); the first peak might be consistent with zygotic expression of the PIWI-class transcripts; the second peak was detected at the late larval stage (Figure 6D). Both peaks coincided with gonad development and formation during embryonic and larval stages in An. gambiae.

\section{Discussion}

Both the siRNA and piRNA pathways are known to be involved in the control of TE activity and gene expression. Here we examined the diversity and abundance of the mixed somatic/germline piRNAs and siRNAs in $A n$. gambiae adult mosquitoes. The vast majority of $A n$. gambiae piRNAs and siRNAs were produced from TEs in all analysed libraries. The An. gambiae TE-derived siRNAs mapped predominantly to class II TEs and piRNAs mapped to class I TEs; this is consistent with the previously published observation in Ae. aegypti [20]. The An. gambiae TE-associated piRNAs showed "ping-pong" sequence signatures and were most likely produced via "ping-pong" dependent mechanism. We found that more than $90 \%$ of the TE-derived 24-30-nt reads were associated with three LTR transposons, gypsy, copia and Pao-Bel that represent the most transcriptionally active TE-piRNAs in mosquito species (this study; [20,32]). Among them, gypsy was the most abundant source of endogenous piRNAs that mapped predominantly to the truncated or Solo-LTR copies in the An. gambiae genome. Overall, our results showed that TE-piRNA production in An. gambiae shared the basic features of piRNA biogenesis, which is conserved among insect species $[15,20,32,52]$. It is worth noting that the relationship between the abundance of TE-piRNAs and piRNA-mediated silencing is complex and likely depends on both transcriptional and posttranscriptional mechanisms of silencing, which might differ among TEs [53]. Interestingly, a number of gypsy insertions are associated with a set of known immune genes in An. gambiae and plausibly influence the expression of the targeted genes [41].

piRNAs are generally produced from a long primary RNA precursors uni- and bidirectionally transcribed from piRNA clusters containing transposon sequence in random orientation [15]. Interestingly, the orientation of the primary piRNA transcript can be regulated throughout development [35]. We found that more than $90 \%$ of clustering endogenous piRNAs were produced by TEs in $A n$. gambiae, similarly to $D$. melagaster. In mosquito species, large differences have been observed in genome size and content of TEs. In contrast to An. gambiae, Ae. agypti has an expansion of the piRNA biogenesis genes and a large TE load that constitutes $47 \%$ of the genome and produces only $19 \%$ of the total piRNAs [32]. Nevertheless, the TE-derived piRNAs represent an important aspect of robust control of TE activities and associated with a low rate of TE remobilization in Ae. agypti [32]. In An. gambiae, we observed a correlation between TE transcription and antisense production of piRNAs similarly to 

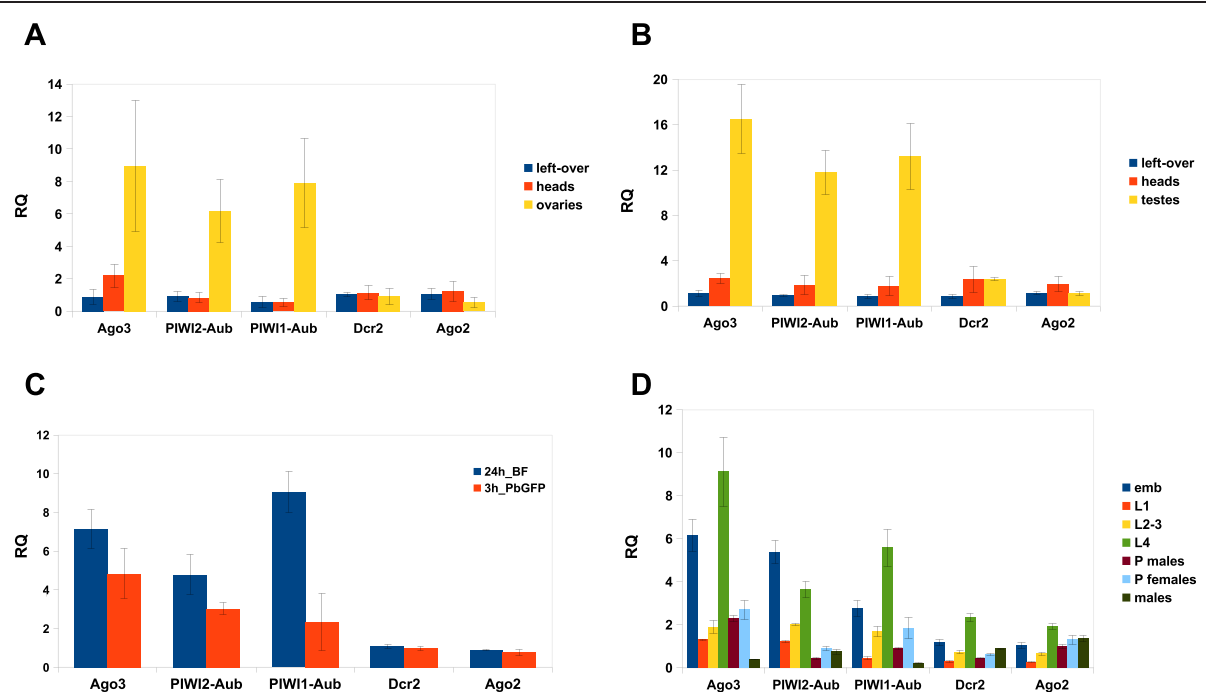

Figure 6 Quantitative RT-PCR analysis of Dcr-2, Ago-2 and PIWI-class transcripts in An. gambiae. (A-B) Transcript expression profiling in adult somatic and germline organs and tissues. Transcript levels were normalized to Rp/19 (endogenous control) and shown as a fold change above the level in one-two day old female (A) and male (B) whole body respectively. (C) Analysis of Dcr-2, Ago-2 and PIWI-class transcripts $3 \mathrm{~h}$ after infectious feeding and $24 \mathrm{~h}$ after regular blood feeding in whole female body. The transcript levels are shown as a fold change above the level in sugar-fed females. (D) Developmental profiling showing the transcript levels in embryos (emb), $1^{\text {st }}$ instar larvae (L1), $2^{\text {nd- }} 3^{\text {rd }}$ instar larvae (L2-3), $4^{\text {th }}$ instar larvae (L4), male and female pupae (P), one-two day old male adults. Transcript levels were normalized to Rp/19 and shown as a fold change above the level in one-two day old female whole body.

Ae. agypti. In addition, the association of clustering piRNAs with TE-derived sequences was statistically significant in An. gambiae, in contrast to Ae. agypti. Curiously, a significant proportion of the endogenous piRNA population was derived from protein coding genes in both $A n$. gambiae and Ae. agypti species (this study, [32]) and mapped predominantly to the sense strand of host genes within gene boundaries in An. gambiae. We showed that a majority of the An. gambiae genic piRNAs were missing the 'ping-pong" sequence signatures and most likely produced via "ping-pong" independent mechanism. Interestingly, the core components of the piRNA biogenesis were not targeted by endogenous siRNAs and piRNAs in the $A n$. gambiae adult mosquitoes, unlike Ae. agypti. Altogether, these findings suggest that some aspects of the piRNA biogenesis and piRNA-mediated regulation might differ between distinct mosquito species.

It has been shown that in the presence of exogenous and endogenous genetic elements the production of endo-piRNAs and endo-siRNAs were induced in Drosophila and mosquitoes [32,49,54]. Furthermore, arbovirus infection of cells derived from natural vector $A$. albopictus and Ae. aegypti induced de novo production of virus-specific small RNAs and endogenous piRNAs mapped to TEs [9,19,20,30-32]. An. gambiae is a principal malaria vector in Sub-saharan Africa of a high medical importance. We found that blood feeding induced de novo production of genic piRNAs and piRNAs mapped to DNA transposons that became a source of additional piRNAs in adult An. gambiae females. However, in the presence of $P$. berghei parasite we did not detect de novo produced endogenous piRNAs. An. gambiae is also known as a primary vector of the alphavirus O'nyong-yong. It has been shown that antiviral defence against systemic O'nyong-yong infection in An. gambiae requires Ago-2 activity for efficient RNA silencing [8]. Yet the detailed analysis of small RNA repertoire in the infected $A n$. gambiae mosquitoes using deep sequencing has been not performed so far.

Mosquito PIWI family members exhibit both germline specific expression restricted to gonads and somatic expression resulting in functional RNAi activity $[19,20]$. In our study, the core components of the siRNA and piRNA pathways were up-regulated after blood feeding in $A n$. gambiae female guts and gonads respectively. Recently, the expression profiles of PIWI-class transcripts have been reported in the closely related An. stephensi species [55]. Both Anopheles species showed gonad specific enrichment for PIWI-class transcripts and up-regulation of their expression levels after blood feeding (this study; [55]).

TE-dependent chromosomal rearrangements are known to be involved in the shaping of genome landscapes and creating new gene blocks that influence ecological adaptation, plasticity, behaviour and vector capacity in the panAfrican malaria vector, An. gambiae [56]. In the genome of An. gambiae the sex chromosome and autosomes have different genome landscapes and distinct enrichments by TEs. The distribution of TEs is higher overall 
near centromeres in the euchromatic regions, near the telomeres and in addition differs by chromosome arms [27]. The highest TE density is associated with the fastest evolving chromosome $\mathrm{X}$, where the majority of fixed inversions were found. Importantly, the $\mathrm{X}$ chromosome is enriched for genes that are known to play a role in reproductive isolation and specification in Anopheles. A large number of autosomal paracentric polymorphic inversions has been reported on the $2 \mathrm{R}$ chromosome, which is associated overall with the lowest transposon and repeat densities [27]. Interestingly, the majority of the $2 \mathrm{R}$ arm inversions are associated with clustered segmental duplications and insertions of remnants of repetitive sequences including class I and II transposable elements [56,57]. In addition, it has been reported that the breakpoints for the chromosomal inversion 2La appear to be enriched for LTR transposons [41]. These inversions are associated with population structure, conferring ecological adaptation and altering mosquito adaptive fitness [27,58]. Further study is required to determine the precise role of the endogenous siRNAs and piRNAs in the evolution of regulatory and functional genome landscape of $A n$. gambiae. Chromosomal aberration with a deleterious effect on viability and reproduction could be introduced artificially to reduce the size of the vector population, therefore representing a potential vector control strategy based on manipulation of the An. gambiae genome and fitness.

\section{Conclusion}

We analysed the endogenous siRNA and piRNA populations in the African malaria mosquito An. gambiae. Like Ae. agypti, vast majority of An. gambiae piRNAs and siRNAs were produced by TEs from class I and class II transposons respectively. The most abundant An. gambiae TE-associated piRNAs were 26-27-nt in length and most likely produced by a "ping-pong" dependent mechanism; whereas the majority of genic piRNAs were 29-nt in length and were missing the "ping-pong" sequence signatures. Vast majority of the detected piRNAs were produced from TE-associated clusters in An. gambiae, similarly to $D$. melanogaster. Overall, TE-siRNAs and TEpiRNAs were expressed at much higher levels than genic siRNAs and piRNAs. The main components of the siRNA and piRNA biogenesis were under avoidance of being targeted by endogenous siRNAs and piRNAs in adult An. gambiae mosquitoes. Importantly, An. gambiae adult gonads were highly enriched by PIWI-class transcripts indicating the existence of a conserved mechanism, which controls the expression and function of PIWI-class genes in mosquitoes. In addition, the PIWIclass transcripts and two core components of the siRNA pathway, Dcr-2 and Ago-2 were up-regulated after regular and infectious blood feeding in the Anopheles female body and infected female guts respectively. Moreover, blood feeding and P. berghei infection induced de novo production of genic piRNAs that became a source of additional piRNAs in adult females. The mild changes in the expression levels of endogenous siRNAs and piRNAs were observed in response to blood feeding and $P$. berghei infection, which might represent a general response to biotic stress.

\section{Methods}

\section{Mosquito rearing and sample collection}

An. gambiae G3 strain was reared and maintained in humidified chambers at $28^{\circ} \mathrm{C}$ with a $12 \mathrm{~h}$ light/dark cycle. Mosquito samples for quantitative real-time PCR (qRT-PCR) profiling were prepared from ten-fifteen individuals. One-two days old adult whole bodies, heads, adult gonads (testes and ovaries) and leftover were collected from sugar-fed female and male mosquitoes. Mosquito feeding on anaesthetized PbGFPCON-infected mouse and on a regular mouse was performed as described in [33]. Three and twenty-four hours post regular and infectious blood feeding, whole bodies, heads, ovaries, guts and left-over were collected from blood-fed and P. berghei-infected four-five and six day old female mosquitoes respectively. Sugar-fed mosquitoes were used as a control. Developmental profiling was performed on a mixture of the fertilized eggs (0-24 hours after oviposition), a mixture of early larval (L1), intermediate (L2-3) and late larval stages (L4), female and male pupae, one-two day old adult female and male mosquitoes.

\section{Small RNA analysis}

To identify endogenously produced siRNAs and piRNAs in An. gambiae adult females we used six small RNA libraries reported in ([33], GEO accession number GSE50396). The ncPRO-seq analysis pipeline was used to filter out known rRNAs, tRNAs, snRNAs, snoRNAs and miRNAs [59]. The remaining reads were mapped to An. gambiae AgamP3.8 PEST genome assembly (VectorBase) using Bowtie program allowing two mismatches. For the repetitive and mobile elements associated small RNA analysis, all TEs were retrieved from the EnsemblMetazoa database. The sense and antisense small RNA quantifications were performed with a pipeline of custom developed Python scripts. Uniquely and multiply mapped 21-nt reads and 24-30-nt reads were analysed independently and normalized by their read numbers against genomic locations. The raw and normalized read frequency of small RNA reads derived from repetitive elements and TEs or coding genes are listed in Additional file 2: Table S2, Additional file 3: Table S3, Additional file 10: Table S4 and Additional file 8: Table S6, respectively. 
IGV2.3.31 viewer was used to visualize sequence reads mapped to the reference genome.

The overlap pair analysis was performed using collapsed datasets obtained for sugar-fed mosquitoes allowing two mismatches. Quantification of the 21-nt and 24-30-nt paired reads was performed using a pipeline of custom developed Python scripts available upon request. The following pair arrangements between $5^{\prime}$ ends of the reads and their neighbour reads on the opposite strand were analysed for siRNA pairs (21-nt reads in length with the 3 '-end 2-nt overhangs, offset +19 -nt) and for piRNAs (24-30-nt read length; offset in $5^{\prime}$-nucleotides $0 \mathrm{nt} ;+10$ nt or $-(16-18) \mathrm{nt}$ as described in $[15,16]$. Five reads cut-off was used for the paired sense and antisense strands mapping and the read numbers were then normalized for each data set. The piRNA cluster analysis was performed as described in $[15,16]$. We used a $5-\mathrm{kb}$ window across the An. gambiae genome to identify all regions with frequencies greater than one siRNA or piRNA/kb. Only uniquely mapped 24-30-nt reads were considered for the further analysis with a cut-off of 10 reads and more (Additional file 7: Table S5). Sequence compilations for 21-nt and 24-30-nt reads based on relative nucleotide frequency per each position across the reads mapped to sense and antisense strands, separately, were generated using WebLogo3.3. For all WebLogos, libraries from two replicates obtained from sugar-fed females were collapsed. The DESeq2.6 package was used to quantify and to assess differential expression of 21-nt and 24-30-nt reads, which was considered as significant at $p<0.05$ (shown in Additional file 8: Table S6).

\section{Plasmid construction and RNA-based silencing}

RNA interference was used to silence gene expression in adult female mosquitoes. Ago-3 (AGAP008862), PIWI2/ Aub (AGAP009509) and PIWI1/Aub (AGAP011204) fragments were PCR-amplified from a cDNA ( $A n$. gambiae G3 strain) and cloned into pLL110 vector carrying two T7 promoters described in [60]. Dcr-2 (AGAP012289): EcoRI-HindIII 512-bp fragment was subcloned from the 71B02 clone of the Gateway (Invitrogen) immune library described in [61] into the pLL110. The pLL110-Ago-2 previously described in [33]. The following PCR primers were used, Ago-3 forward 5'-GTCAAACATGTACCGCCGTGTG-3'; Ago-3 reverse primer: 5'-CCCCATGATCTGTGGCATTGAC-3'; PIWI2/Aub forward primer: 5'-GCAAACCTCCCCCGAA AGCC-3'; PIWI2/Aub reverse primer 5'-CGACACCACG CACATGATCATC-3'; PIWI1/Aub forward primer: 5'GCGACAAGTCGCTCTCGTACGG-3', PIWI1/Aub reverse primer: 5'- GTACTGGCAGACAGCCGGTAC-3'. Double-stranded RNAs were prepared as described in [60]. One-day post-emerged $\mathrm{CO}_{2}$-anaesthetized mosquito females were injected intrathoracically with $0.6 \mu \mathrm{g}$ of dsRNA using nano-injector (Nanoject II, Drummond). dsRNA of the lacZ gene was used as a control. Efficacy of RNA silencing on gene expression was analysed $24 \mathrm{~h}$ after dsRNA injection by qRT-PCR.

\section{qRT-PCR}

Gene expression profiling and the efficacy of RNA silencing were assessed by Fast SYBR Green-based qPCR (ABI). Total RNA was isolated using a TRI Reagent (MRC). cDNAs were synthesized from $0.4 \mu \mathrm{g}$ of total RNA samples using random hexamer primers and Revert Aid $\mathrm{H}$ Minus cDNA synthesis kit (Thermo Scientific). For the PCR amplification of gypsy and CR1 transcripts, total RNA samples $(0.4 \mu \mathrm{g})$ were pre-treated with DNAse I enzyme (Thermo Scientific). After inactivation of the enzyme, the RNA samples were used for cDNA synthesis as described above; no reverse transcriptase controls were used to access for genomic DNA contamination in the DNAse I pre-treated RNA samples. qPCR was performed using the following primers: $D c r-2$ forward primer: 5'-GCGAAGGCCAGGTAATTATCTG-3', Dcr-2 reverse primer: 5'-GACATTCGTCGAACACGAT CA-3'; Ago-2 forward primer 5'-ATGCTCAAGATCAACG CCAAA-3', Ago-2 reverse primer 5'-TGAGCGGGTGCGT AACGT-3'; Ribosomal protein L19 (RpL19) forward primer 5'-CCAACTCGCGACAAAACATTC-3', RpL19 reverse primer 5'-ACCGGCTTCTTGATGATCAGA-3'; Ago-3 forward primer 5'-CATAAGGTAATGCGCGATATTGC-3', Ago-3 reverse primer 5'-CGGCTTCATTTTTGTTCACAT TC-3'; PIWI2/Aub forward primer: 5'-GCGCTCCGATT TCAAAATGA-3', PIWI2/Aub reverse primer 5'-CGTTTC CAGCCGTTCGATA-3'; PIWII/Aub forward primer: 5'GCCGCAGATCCTTTATTTGC-3', PIWII/Aub reverse primer: 5'-GGTCGGCCCGATCGTT-3'; AGAP003387 forward primer: 5'-AAATCAGTGCGTGTGTCCGAAC-3', reverse primer: 5'- GCGATGGGTCGTTTCCTTACGGTG3'; AGAP001052 forward primer: 5'-CCACTCAAGTTTAT GTGGTCTATGGA-3', reverse primer: 5'-TCAGATGATG ATTGACCTCGTAGAA-3'; gypsy (GYPSY10-I_AG) forward primer: 5'-CCAGATGACTCGAAATACGATAGC-3', gypsy (GYPSY10-I_AG) reverse primer: 5'-GTTTAGCGGTTTTG CTTTCAAAG-3'; CR1-9_AG forward primer: 5'-TCGAC TCCTTCCATGGCAAT-3', CR1-9_AG reverse primer: 5'AAGCAGACACCGCTGATGGT-3'. RplL19 was used as an endogenous control to normalize the transcript levels. PCR reactions were performed on an OneStep Plus thermocycler (ABI), according to the manufacturer's protocol. Each measurement was derived from three independent biological replicates for all expression profile analyses performed in this study. Relative quantification of gene expression was performed using the comparative $\mathrm{Ct}(\Delta \Delta \mathrm{Ct})$ method. 


\section{Ethics statement}

All experimental procedures on mice were performed in accordance with the national animal protection law (Landesamt für Gesundheit und Soziales (LAGeSo)) and approved by the committee for animal use and protection (LAGeSo permit number: $\mathrm{H}$ 0027/12).

\section{Additional files}

Additional file 1: Table S1. Small RNA size distribution and abundance in An. gambiae libraries.

Additional file 2: Table S2. Abundance of small RNAs from An. gambiae libraries mapped to TES.

Additional file 3: Table S3. Abundance of small RNAs from An. gambiae libraries mapped to An. gambiae coding genes.

Additional file 4: Figure S1. Frequency and distribution of 21-nt and 24-30-nt reads mapped to the most abundant NLTR-transposons, DNA transposons and specific coding genes.

Additional file 5: Figure S2. aPCR-based measurement of the transcript levels (the most representative TEs and coding genes) in Dcr-2, Ago-2 and PIWI silenced mosquitoes.

Additional file 6: Figure S3. Expression profiling of the core components of the siRNA and piRNA pathways and the most representative coding genes after regular and infectious blood feeding in An. gambiae females.

Additional file 7: Table S5. The siRNA and piRNA cluster analysis in the genome of An. gambiae.

Additional file 8: Table S6. Differential expression analysis of siRNAs (21-nt) and piRNAs (24-30-nt) in Anopheles females $3 \mathrm{~h}$ after regular and infectious blood feeding. Fold-changes in the expression levels are shown as a ratio of blood-fed (BF) to sugar-fed (SF) mosquitoes or as a ratio of infected (PbGFP) to non-infected blood-fed mosquitoes (BF).

Additional file 9: Figure S4. Expression profiling of the core components of the siRNA and piRNA pathways in sugar and blood-fed An. gambiae females.

Additional file 10: Table S4. The top abundant genes producing endogenous siRNAs and piRNAs in An. gambiae libraries (two collapsed replicate).

\section{Abbreviations}

siRNA: small interfering RNA; piRNA: PIWI-interacting RNA; TE: Transposable element; ncRNA: non-coding RNA; RISC: RNA-induced silencing complex; Aub: Aubergine; PIWI: P-element induced wiped testis; Ago: Argounate; Dcr: Dicer; LTR: Long terminal repeat; NLTR: Non-long terminal repeat; MITE: Miniature inverted transposable element; SINE: Short interspersed nuclear element; cis-NAT: cis-natural antisense transcript; nt: Nucleotide; kb: Kilobase; h: Hour.

\section{Competing interests}

The authors declare that they have no competing interests.

\section{Authors' contributions}

IB designed the project, TYe performed bioinformatics analysis. IB conducted experimental work, analysed and interpreted data, oversaw the bioinformatics analysis and wrote the manuscript. Both authors read and approved the final manuscript.

\section{Acknowledgement}

We thank E. Levashina and Dpt. Vector Biology for sharing department facilities, D. Tschierske, L. Spohr, S. Koppitz and H. Krueger for maintaining mosquito colonies and technical assistance with mouse blood feeding. We would like to acknowledge M.-C. Saleh and R. J. Clayton for helpful discussion. We thank G. Volohonsky for reading of this manuscript and providing critical comments. Sequencing analysis was performed by the IGBMC Microarray and Sequencing platform and supported by the FG National Infrastructure, funded as a part of the "Investissements d'Avenir" program managed by the Agence Nationale pour la Recherche (ANR-10-INBS-0009). This work was supported by the European Community's Seventh Framework Programme (FP7/2007-2013) under grant agreements no. 242095-EVIMalar and no. 223601-MALVECBLOK.

\section{Author details}

${ }^{1}$ Department of Vector Biology, Max Planck Institute for Infection Biology (MPIIB), Berlin 10117, Germany. ${ }^{2}$ Microarrays and deep sequencing platform, Institut de Génétique et de Biologie Moléculaire et Cellulaire (IGBMC), IIlkirch Cedex 67404, France.

Received: 16 November 2014 Accepted: 6 March 2015

Published online: 10 April 2015

\section{References}

1. Kim VN, Han J, Siomi MC. Biogenesis of small RNAs in animals. Nat Rev Mol Cell Biol. 2009;10:126-39.

2. Ghildiyal M, Zamore PD. Small silencing RNAs: an expanding universe. Nat Rev Genet. 2009;10:94-108.

3. Malone C, Hannon G. Small RNAs as guardians of the genome. Cell. 2009;136(4):656-68.

4. van Rij RP, Berezikov E. Small RNAs and the control of transposons and viruses in Drosophila. Trends Microbiol. 2009;17:163-71.

5. Senti K, Brennecke J. The piRNA pathway: a fly's perspective on the guardian of the genome. Trends in Genetics: TIG. 2010;26:499-509.

6. Rozhkov N, Hammell M, Hannon G. Multiple roles for Piwi in silencing Drosophila transposons. Genes Dev. 2013;27:400-12.

7. Li C, Vagin W, Lee $S, X u J, M a ~ D, X i ~ H$, et al. Collapse of germline piRNAs in the absence of Argonaute3 reveals somatic piRNAs in flies. Cell. 2009:137:509-21.

8. Keene K, Foy B, Sanchez-Vargas I, Beaty BJ, Blair C, Olson KE. RNA interference acts as a natural antiviral response to O'nyong-nyong virus (Alphavirus; Togaviridae) infection of Anopheles gambiae. Proc Natl Acad Sci U S A. 2004;101:17240-5.

9. Campbell CL, Keene KM, Brackney DE, Olson KE, Blair CD, Wilusz J, et al. Aedes aegypti uses RNA interference in defense against Sindbis virus infection. BMC Microbiol. 2008;8:47.

10. Siomi MC, Sato K, Pezic D, Aravin AA. PIWl-interacting small RNAs: the vanguard of genome defence. Nat Rev Mol Cell Biol. 2011;12:246-58.

11. Sato K, Siomi MC. Piwi-interacting RNAs: biological functions and biogenesis Essays Biochem. 2013;54:39-52.

12. Ross RJ, Weiner MM, Lin H. PIWI proteins and PIWl-interacting RNAs in the soma. Nature. 2014;505:353-9.

13. Gunawardane LS, Saito K, Nishida KM, Miyoshi K, Kawamura Y, Nagami T, et al. A slicer-mediated mechanism for repeat-associated siRNA $5^{\prime}$ end formation in Drosophila. Science (New York, NY). 2007;315:1587-90.

14. Czech B, Hannon GJ. Small RNA sorting: matchmaking for Argonautes. Nature reviews. Genetics. 2011;12:19-31.

15. Brennecke J, Aravin AA, Stark A, Dus M, Kellis M, Sachidanandam R, et al. Discrete small RNA-generating loci as master regulators of transposon activity in Drosophila. Cell. 2007;128:1089-103.

16. Lau NC, Robine N, Martin R, Chung W-JJ, Niki Y, Berezikov E, et al. Abundant primary piRNAs, endo-siRNAs, and microRNAs in a Drosophila ovary cell line. Genome Res. 2009:19:1776-85.

17. Schnettler E, Donald CL, Human S, Watson M, Siu RWC, McFarlane M, et al. Knockdown of piRNA pathway proteins results in enhanced Semliki Forest virus production in mosquito cells. J Gen Virol. 2013;94:1680-9.

18. Leger $\mathrm{P}$, Lara $\mathrm{E}$, Jagla B, Sismeiro O, Mansuroglu Z, Coppee JY, et al. Dicer-2- and Piwi-mediated RNA interference in Rift Valley fever virus-infected mosquito cells. J Virol. 2013;87:1631-48.

19. Morazzani EM, Wiley MR, Murreddu MG, Adelman ZN, Myles KM. Production of Virus-Derived Ping-Pong-Dependent piRNA-like Small RNAs in the Mosquito Soma. PLoS Pathog. 2012;8(1):e1002470.

20. Vodovar N, Bronkhorst AW, van Cleef KWR, Miesen P, Blanc H, van Rij RP, et al. Arbovirus-Derived piRNAs exhibit a ping-pong signature in mosquito cells. PLoS One. 2012;7(1):e30861.

21. Bronkhorst AW, van Rij RP. The long and short of antiviral defense: small RNA-based immunity in insects. Current Opinion in Virology. 2014;7C:19-28.

22. Wua Q, Luoa Y, Lua R, Laub N, Laic EC, Lia WX, et al. Virus discovery by deep sequencing and assembly of virus-derived small silencing RNAs. Proc Natl Acad Sci. 2010;107:1606-11. 
23. Houwing S, Kamminga LM, Berezikov E, Cronembold D, Girard A, van den Elst $\mathrm{H}$, et al. A role for Piwi and piRNAs in germ cell maintenance and transposon silencing in zebrafish. Cell. 2007;129:69-82.

24. Fernandez-Medina RD, Ribeiro JMC, Carareto CMA, Velasque L, Struchiner CJ. Losing identity: structural diversity of transposable elements belonging to different classes in the genome of Anopheles gambiae. BMC Genomics. 2012;13:272

25. Miller WJ, McDonald JF, Pinsker W. Molecular domestication of mobile elements. Genetica. 1997;100:261-70.

26. Kapitonov W, Jurka J. A universal classification of eukaryotic transposable elements implemented in Repbase. Nature reviews. Genetics. 2008;9:411-2.

27. Holt RA, Subramanian GM, Halpern A, Sutton GG, Charlab R, Nusskern DR, et al. The genome sequence of the malaria mosquito Anopheles gambiae. Science (New York, NY). 2002;298:129-49.

28. Arensburger P, Megy K, Waterhouse RM, Abrudan J, Amedeo P, Antelo B, et al. Sequencing of Culex quinquefasciatus establishes a platform for mosquito comparative genomics. Science (New York, NY). 2010;330:86-8.

29. Fernandez-Medina RD, Struchiner CJ, Ribeiro JM. Novel transposable elements from Anopheles gambiae. BMC Genomics. 2011;12:260.

30. Hess AM, Prasad AN, Ptitsyn A, Ebel GD, Olson KE, Barbacioru C, et al. Smal RNA profiling of Dengue virus-mosquito interactions implicates the PIWI RNA pathway in anti-viral defense. BMC Microbiol. 2011;11:45.

31. Adelman ZN, Anderson MA, Liu M, Zhang L, Myles KM. Sindbis virus induces the production of a novel class of endogenous siRNAs in Aedes aegypti mosquitoes. Insect Mol Biol. 2012;21:357-68.

32. Arensburger $\mathrm{P}$, Hice $\mathrm{RH}$, Wright JA, Craig NL, Atkinson PW. The mosquito Aedes aegypti has a large genome size and high transposable element load but contains a low proportion of transposon-specific piRNAs. BMC Genomics. 2011;12:606.

33. Biryukova I, Ye T, Levashina E. Transcriptome-wide analysis of microRNA expression in the malaria mosquito Anopheles gambiae. BMC Genomics. 2014;15:557

34. Betel D, Sheridan R, Marks DS, Sander C. Computational analysis of mouse piRNA sequence and biogenesis. PLoS Comput Biol. 2007;3(11):e222.

35. Wei C, Salichos L, Wittgrove CM, Rokas A, Patton JG. Transcriptome-wide analysis of small RNA expression in early zebrafish development. RNA (New York, NY). 2012;18:915-29.

36. Wen J, Mohammed J, Bortolamiol-Becet D, Tsai H, Robine N, Westholm JO, et al. Diversity of miRNAs, siRNAs, and piRNAs across 25 Drosophila cell lines. Genome Res. 2014;24:1236-50.

37. Chung WJ, Okamura K, Martin R, Lai EC. Endogenous RNA Interference provides a somatic defense against drosophila transposons. Curr Biol. 2008;18:795-802.

38. Vagin W, Sigova A, Li C, Seitz H, Gvozdev V, Zamore PD. A Distinct small RNA pathway silences selfish genetic elements in the germline. Science. 2006;313:320-4.

39. Czech B, Malone CD, Zhou R, Stark A, Schlingeheyde C, Dus M, et al. An endogenous small interfering RNA pathway in Drosophila. Nature. 2008:453:798-802.

40. Ghildiyal M, Seitz H, Horwich MD, Li C, Du T, Lee S, et al. Endogenous siRNAs derived from transposons and mRNAs in Drosophila somatic cells. Science (New York, NY). 2008;320:1077-81.

41. Tubio JM, Tojo M, Bassaganyas L, Escaramis G, Sharakhov IV, Sharakhova MV, et al. Evolutionary dynamics of the Ty3/gypsy LTR retrotransposons in the genome of Anopheles gambiae. PLoS One. 2011;6(1):e16328.

42. Biedler J, Tu Z. Non-LTR Retrotransposons in the african malaria mosquito, Anopheles gambiae: unprecedented diversity and evidence of recent activity. Mol Biol Evol. 2003;20:1811-25.

43. Hoa NT, Keene KM, Olson KE, Zheng L. Characterization of RNA interference in an Anopheles gambiae cell line. Insect Biochem Mol Biol. 2003:33:949-57.

44. Campbell CL, Black WC, Hess AM, Foy BD. Comparative genomics of small RNA regulatory pathway components in vector mosquitoes. BMC Genomics. 2008;9:425.

45. Saito $K$, Inagaki S, Mituyama $T$, Kawamura $Y$, Ono $Y$, Sakota E, et al. A regulatory circuit for piwi by the large Maf gene traffic jam in Drosophila. Nature. 2009:461:1296-9.

46. Robine N, Lau NC, Balla S, Jin Z, Okamura K, Kuramochi-Miyagawa S, et al. A broadly conserved pathway generates $3^{\prime} U T R$-directed primary piRNAs. Curr Biol. 2009;2066-2076:19
47. Moy RH, Cole BS, Yasunaga A, Gold B, Shankarling G, Varble A, et al. Stem-Loop Recognition by DDX17 Facilitates miRNA Processing and Antiviral Defense. Cell. 2014;158:764-77

48. Ishizuka A, Siomi MC, Siomi H. A Drosophila fragile $X$ protein interacts with components of RNAi and ribosomal proteins. Genes Dev. 2002;16:2497-508.

49. Shpiz S, Ryazansky S, Olovnikov I, Abramov Y, Kalmykova A. Euchromatic transposon insertions trigger production of novel pi- and endo-siRNAs at the target sites in the Drosophila germline. PLoS Genet. 2014;10(2):e1004138.

50. Marinotti O, Calvo E, Nguyen QK, Dissanayake S, Ribeiro JM, James AA. Genome-wide analysis of gene expression in adult Anopheles gambiae. Insect Mol Biol. 2006;15:1-12.

51. Anders S. Analysing RNA-Seq data with the DESeq package. Mol Biol. 2010;43(4):1-17.

52. Kawaoka S, Hayashi N, Suzuki Y, Abe H, Sugano S, Tomari Y, et al. The Bombyx ovary-derived cell line endogenously expresses PIWI/PIWI-interacting RNA complexes. RNA. 2009;15:1258-64.

53. Kelleher ES, Barbash DA. Analysis of piRNA-mediated silencing of active TEs in Drosophila melanogaster suggests limits on the evolution of host genome defense. Mol Biol Evol. 2013;30:1816-29.

54. Olovnikov I, Ryazansky S, Shpiz S, Lavrov S, Abramov Y, Vaury S, et al. De novo piRNA cluster formation in the Drosophila germ line triggered by transgenes containing a transcribed transposon fragment. Nucleic Acids Res. 2013:41(11):5757-68.

55. Macias V, Coleman M, Bonizzoni M, James AA. piRNA pathway gene expression in the malaria vector mosquito Anopheles stephensi. Insect Mol Biol. 2014;23(5):579-86.

56. Xia A, Sharakhova MV, Leman SC, Tu Z, Bailey JA, Smith CD, et al. Genome landscape and evolutionary plasticity of chromosomes in malaria mosquitoes. PloS One. 2010;5:e10592.

57. Coulibaly MB, Lobo NF, Fitzpatrick MC, Kern M, Grushko O, Thaner DV, et al. Segmental duplication implicated in the genesis of inversion $2 \mathrm{Rj}$ of Anopheles gambiae. PLoS One. 2007;2:e849.

58. Gray EM, Rocca KA, Costantini C, Besansky NJ. Inversion 2La is associated with enhanced desiccation resistance in Anopheles gambiae. Malar J. 2009;8:215.

59. Chen C-JJ, Servant N, Toedling J, Sarazin A, Marchais A, Duvernois-Berthet E, et al. ncPRO-seq: a tool for annotation and profiling of ncRNAs in sRNA-seq data. Bioinformatics (Oxford, England). 2012;28:3147-9.

60. Blandin S, Shiao S-HH, Moita LF, Janse CJ, Waters AP, Kafatos FC, et al. Complement-like protein TEP1 is a determinant of vectorial capacity in the malaria vector Anopheles gambiae. Cell. 2004;116:661-70.

61. Frolet C, Thoma M, Blandin S, Hoffmann JA, Levashina EA. Boosting NF-kappaB-dependent basal immunity of Anopheles gambiae aborts development of Plasmodium berghei. Immunity. 2006;25:677-85.

\section{Submit your next manuscript to BioMed Central and take full advantage of:}

- Convenient online submission

- Thorough peer review

- No space constraints or color figure charges

- Immediate publication on acceptance

- Inclusion in PubMed, CAS, Scopus and Google Scholar

- Research which is freely available for redistribution 